\title{
MINIMIZATION OF ORDERED PSEUDO KRONECKER DECISION DIAGRAMS
}

\author{
Per Lindgren \\ Division of Computer Engineering \\ Luleå University of Technology \\ 97187 Luleå, Sweden \\ pln@sm.luth.se
}

\author{
Rolf Drechsler \\ Bernd Becker \\ Institute of Computer Science \\ Albert-Ludwigs-University \\ 79110 Freiburg im Breisgau, Germany \\ \{drechsle/becker\}@informatik.uni-freiburg.de
}

\begin{abstract}
The introduction of Decision Diagrams (DDs) has brought new means towards solving many of the problems involved in digital circuit design. Compactness of the representation is one key issue. Ordered Pseudo Kronecker Decision Diagrams (OPKDDs) together with the use of complemented edges is known to offer the most general ordered, read-once $D D$ representation at the bit-level, hence OPKDDs hold all minimal sized bit-level ordered $D D$ s for a given function. This representation allows us to trade-off diagram canonicity against compactness. Ternary-OPKDDs (TOPKDDs) implicitly holds all OPKDDs for a given variable order. We state the canonicity criteria for TOPKDDs having complemented edges and develop an efficient sifting based method for their minimization. Furthermore, a heuristic minimization algorithm for $O P K D D s$ is devised, utilizing the redundancies of Ternary-OPKDDs (TOPKDDs). Experiments on a set of MCNC benchmarks confirm the potential compactness of $O P K D D s$ and demonstrate the efficiency of the proposed heuristics.
\end{abstract}

\section{INTRODUCTION}

The introduction of Decision Diagrams (DDs) has brought new means towards solving many of the problems involved in digital circuit design. Diagram size (compactness) is a sought after property, e.g., in DD based logic synthesis applications. A number of different approaches have been proposed, either utilizing the inherent structure of the DD (e.g., as in [18] for LUT-FPGA mapping and in [19] to derive pass-transistor circuits) or as a starting point for decomposition (e.g., as in [22]). It is known that the size (complexity) of the DD representation may vary from linear to exponential under different variable orderings and decomposition types applied.

In [1] it is shown that three decomposition types together with complemented edges, cover all bit-level decompositions. Therefore, Ordered Pseudo Kronecker Decision Diagrams (OPKDDs) offer the most general bit-level, ordered readonce DD representation of switching functions. In an OPKDD we can for each node in the diagram chose decomposition type \{Shannon, positive Davio or negative Davio\} arbitrarily. In that, we give up the property of canonicity in order to reduce the diagram size.

The complexity of the OPKDD representation is dependent on both the ordering variables and the decomposition type applied to each node. The number of possible OPKDDs for a function of $n$ variables is $n ! 3^{2^{n}-1}$ [17], implying the need for heuristic minimization methods.

A Ternary Ordered Pseudo Kronecker Decision Diagram (TOPKDD) (also known as canonical Exor Ternary Decision Diagram (ETDD) [17]) is a redundant representation implicitly holding all OPKDDs for a function under a given variable ordering. We give the conditions under which TOPKDDs having complemented edges offer a canonical representation and show that local variable exchange on the diagram can be efficiently conducted while preserving canonicity. These properties enable us to devise mechanisms for their minimization, similar to those known for other DDs, $[10,16,7]$. Furthermore, we show how to utilize the redundancy of TOP. KDDs to derive OPKDDs. By our experiments, the potentiail compactness of OPKDDs is confirmed.

The paper is outlined as follows; Section 2 first gives a background on representation of switching functions using decision diagrams. A sifting based method for minimization of TOPKDDs is presented in Section 3. Furthermore, a heuristic method for OPKDD minimization is proposed in Section 4. We evaluate their performance on a set of MCNC benchmarks in Section 4.1. Finally, the paper is concluded in Section 5 .

\section{ORDERED PSEUDO KRONECKER DECISION DIAGRAMS}

In this section we review the essentials of OPKDDs and highlight some important features.

Let $f_{0}\left(f_{1}\right)$ denote the cofactor of $f$ with respect to $\bar{x}(s x)$ and $f_{2}$ is defined as $f_{2}=f_{0} \oplus f_{1}, \oplus$ being the Exclusive OR (EXOR) operation. A Boolean function $f: B^{n} \rightarrow B$ can then be represented by one of the following formulae:

$$
\begin{aligned}
& f=\bar{x} f_{0} \oplus x f_{1} \quad \text { Shannon }(S h) \\
& f=f_{0} \oplus x f_{2} \quad \text { positive Davio }(p D) \\
& f=f_{1} \oplus \bar{x} f_{2} \quad \text { negative Davio }(n D)
\end{aligned}
$$


Consider a rooted, Directed Acyclic Graph (DAG) having terminals 0 and 1 and non-terminals (internal nodes) $G$. Each node in $G$ is marked with a decomposition variable $x$, a decomposition type $\{S h, p D, n D\}$ and edges to cofactors $\left\{\left(f_{0}, f_{1}\right),\left(f_{0}, f_{2}\right),\left(f_{1}, f_{2}\right)\right\}$ according to decomposition type. In the following we assume each variable to occur only once on each path and in the same order for all paths (i.e., the DAG is "ordered"). Nodes having the same decomposition variable are considered to be at the same level in the diagram. Diagram levels are enumerated from root (top level) towards terminals (bottom level). Furthermore, we apply the following rules of "reduction":

- There exist no two nodes expressing the same function (i.e., no two nodes are graph-isomorphic).

- There exist no Shannon ( $S h$ ) node having $f_{0}=f_{1}$, and no Davio nodes $\left(p D\right.$ and $n D$ ) having $f_{2}=0$.

This defines a Ordered Pseudo Kronecker Decision Diagram (OPKDD) [18]. By applying restrictions other DDs can be defined, e.g., Ordered Binary Decision Diagrams (OBDDs) [3] (having Shannon ( $S h$ ) nodes exclusively), and Ordered Kronecker Functional Decision Diagrams (OKFDDs) [8, 4] (where the decomposition type $\{S h, p D, n D\}$ is fixed for each variable). A single node is used to represent both $f$ and $\bar{f}$, the latter identified by a complement attribute on the incoming edge $[12,2]$. For a given ordering a reduced OBDD offers a canonical representation of the function, under some restrictions for the use of complemented edges. Only one terminal is to be used (e.g., the 0-terminal), and complementation is only allowed to occur on one of the outgoing edges (e.g., the 1-edge). Canonicity also holds for reduced OKFDDs given an ordering and a Decomposition Type List (DTL) together with some restrictions on the use of complemented edges. However, OPKDDs in their generalized form does not provide such a canonical representation. The DDs described above can be used also to represent multiple-output functions, e.g., by allowing each output function to be rooted arbitrarily in the shared-DD. In the following we assume all DDs to be ordered and reduced if not else stated.

The following applies to Figure 1. Each node is marked with its respective node function, its decomposition type $\{S h, p D, n D\}$ and its decomposition variable $x_{i}$. The line type of an edge $\{$ dashed, whole, dotted $\}$ indicates a connection to a cofactor $\left\{f_{0}, f_{1}, f_{2}\right\},\left(f_{2}=f_{0} \oplus f_{1}\right)$ respectively. For the sake of clarity, edges are sometimes also marked with its corresponding literal. Furthermore, the diagrams are left unreduced, and complemented edges are not applied. Figure 1 together with the examples below highlights some important features.

Example 1 Figure $1($ a) shows an $O B D D$ for the parity function $f\left(x_{1}, x_{2}, x_{3}\right)=\bar{x}_{1} \bar{x}_{2} x_{3} \oplus \bar{x}_{1} x_{2} \bar{x}_{3} \oplus x_{1} \bar{x}_{2} \bar{x}_{3} \oplus x_{1} x_{2} x_{3}$. The $D D$ in Figure 1 (b) (representing the same function as (a)), has a $(p D)$ decomposition for node $f$, (Sh) decompositions for nodes $f_{0}$ and $f_{00}$ and $a(n D)$ decomposition for $f_{01}$.

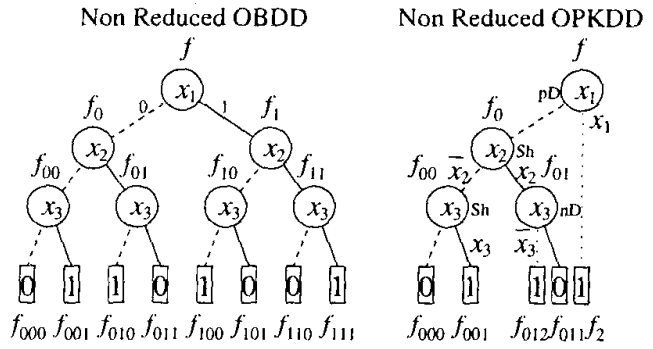

(a)

(b)

Figure 1: Decision Diagrams.

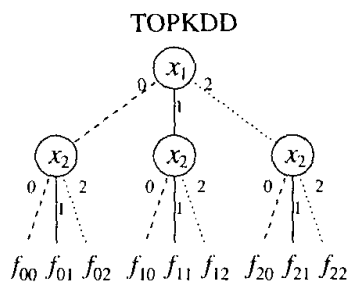

(a)

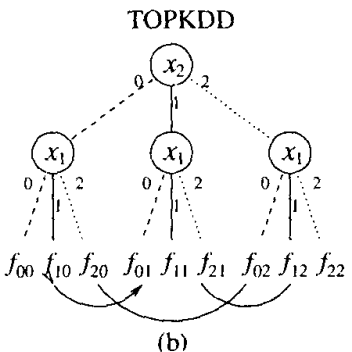

(b)
Figure 2: Local variable exchange on an TOPKDD.

As the decomposition types are mixed $\{S h, p D, n D\}$ and not fixed for each variable (e.g., $\left.x_{2}\right)$ it is an $O P K D D$.

\section{TERNARY ORDERED PSEUDO KRONECKER DECISION DIAGRAMS}

A Ternary Ordered Pseudo Kronecker Decision Diagram (TOPKDD) (also known as canonical Exor Ternary Decision Diagram (ETDD) [17]) is a DD according to Section 2, with the following exceptions; each node in $G$ has three outgoing edges to cofactors $f_{0}, f_{1}$ and $f_{2}=f_{0} \oplus f_{1}$. The same reduction rules as for OBDDs apply. Although a TOPKDD offers a canonical representation (under a given variable order) it is a redundant representation. Given two out of the three outgoing edges, we can always derive the third one by a simple EXOR operation, see Section 2.

As for other DDs, the complexity of the representation depends heavily on the variable order. Heuristic methods have proven successful solving this problem for both OBDDs and OKFDDs. The most efficient methods for OBDD minimization are based on local variable exchange, "sifting" two neighboring levels in the diagram $[10,16,7]$. The key to their success is the property that such a level exchange is indeed a local operation. I.e., nodes belonging to levels above and below the sifted levels are unaffected by the operation. Furthermore, the number of nodes on a fixed level is unaffected by the ordering of the nodes above and below this level.

Let us consider the TOPKDDs shown in Figure 2. (a) shows the initial representation while (b) shows the representation after exchanging the neighboring levels, i.e., (a) 
TOPKDD

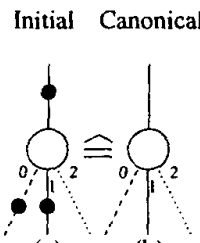

(a) (b)

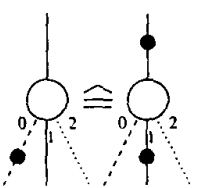
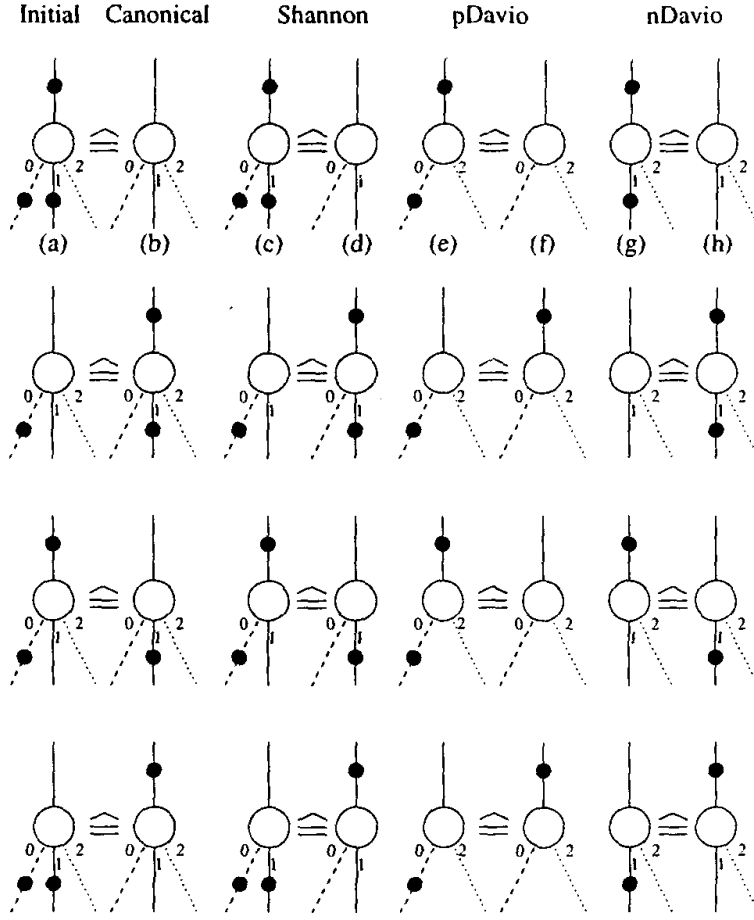

Figure 3: Complemented edges for TOPKDDs.

has variable order $\left(x_{1}, x_{2}\right)$ and (b) has $\left(x_{2}, x_{1}\right)$. We observe that no new cofactors needs to be computed, and that a level exchange can be performed solely by the redirection of edges. The arrows in the figures show the interchange between cofactors applied. The correctness of this may not be obvious, but by simple calculations we prove that this is the case: Consider the three leftmost cofactors of figure (b). The cofactor $f_{00}$ is obviously left unchanged ( $\left.f\right|_{x_{1}=0, x_{2}=0}=\left.f\right|_{x_{2}=0, x_{1}=0}$ ). The next cofactor $f_{10}$ is to be found in (a) by tracing the 1-edge of the top node and the 0 edge of the successor. Now, to the interesting part, cofactor $f_{20}$ (as found in (a) by tracing the 2-edge and 0-edge respectively). We now need to show that $f_{20}=f_{00} \oplus f_{10}$. This becomes obvious as $f_{2}=f_{0} \oplus f_{1}$ (by the definition in Section 2). Applying further co-factorization w.r.t., $x_{2}=0$ preserves the equality (as the functions are the same). Verifying the remaining cofactors can be done accordingly.

\subsection{Complemented Edges}

Complemented edges applied to DDs, have shown not only to reduce diagram sizes but also to improve computational efficiency, $[12,2]$. However, as canonicity of the representation is crucial to many DD applications, we must first make certain that the required conditions are met.

In Figure 3 an edge marked by a filled dot indicates the

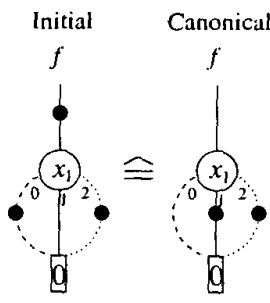

(a) (b)

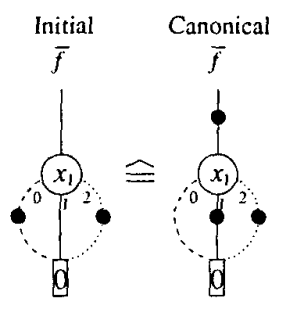

(c) (d)
Figure 4: Canonical TOPKDD representation.

complementation of the corresponding cofactor, (negative polarity). Each row represents the same function, interpreted as TOPKDDs, (shown in the leftmost column), and OPKDDs (shown in the three rightmost columns). To obtain a canonical TOPKDD we have to consistently choose one of the two possible representations for each class of functions (i.e., row). In our case we have chosen the representations marked Canonical. In the following we will prove that evaluation of the diagram is consistent under the proposed transformations:

Consider the top row in Figure 3. The function interpreted as a (non-canonical) Shannon decomposition (c), is $f=\overline{\bar{x} \overline{f_{0}} \oplus x \overline{f_{1}}}=\bar{x} \overline{f_{0}} \cdot x \overline{f_{1}}+\overline{\bar{x} \overline{f_{0}} \cdot \overline{x \overline{f_{1}}}}=\left(x+f_{0}\right)\left(\bar{x}+f_{1}\right)=$ $\bar{x} f_{0}+x f_{1}+f_{0} f_{1}=\bar{x} f_{0}+x f_{1}$, which is obviously correct w.r.t. the canonical representation shown (d). Now consider the (non-canonical) positive Davio representation (e) $f=\overline{\overline{f_{0}} \oplus x f_{2}}=f_{0} \oplus x f_{2}$, which is the function defined by the canonical representation ( $f$ ). From the definition in Section 2 this is equivalent to the Shannon decomposition shown in (d). For clarity we carry out the computation, $f=f_{0} \oplus x f_{2}=f_{0} \oplus x\left(f_{0} \oplus f_{1}\right)=\left(f_{0} \oplus x f_{0}\right) \oplus x f_{1}=$ $\left(\overline{f_{0}} x f_{0}+f_{0}\left(\bar{x}+\overline{f_{0}}\right)\right) \oplus x f_{1}=\bar{x} f_{0} \oplus x f_{1}$. To complete the proof for the top row, let us consider the (non-canonical) negative Davio decomposition (g), $f=\overline{\overline{f_{1}} \oplus \bar{x} f_{2}}=f_{1} \oplus \bar{x} f_{2}$, corresponding to the canonical representation (h).

From the definition in Section 2 this corresponds to the: Shannon decomposition shown in (d). Verifying the remaining cases (rows 2,3 and 4) can be done accordingly.

By this we have given the necessary conditions under which TOPKDDs offer a canonical representation. The rules applied are essentially the same as those used for OBDDs, as the third edge $\left(f_{2}\right)$ neither is used in the canonicity criteria nor is affected by the transformations. Note that the thirid edge $\left(f_{2}\right)$ may or may not be complemented, as determined from its corresponding canonical representation.

Example 2 Consider the TOPKDD representation of function $f=x_{1}$, shown in Figure $4(a)$. By the 3rd-row transformation from Figure 3 we obtain the canonical representation (b). Now consider the function $\bar{f}=\overline{x_{1}}$, shown in Figure 4 (c). The 2nd-row transformation applies and we obtain the canonical representation (d). We observe that the graphs are isomorphic, only the complementation on the incoming edge differs between $f$ and $\bar{f}$. 


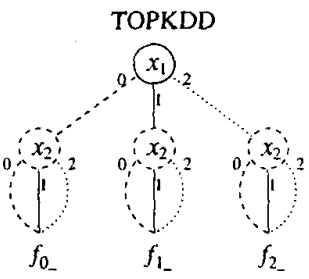

(a)

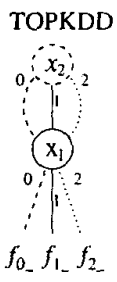

(b)
Figure 5: TOPKDD sifting, changing the number of crosspoint nodes.

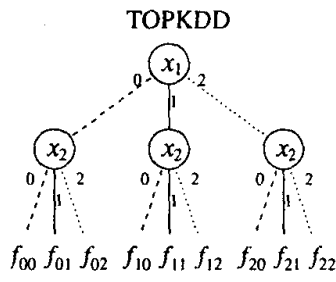

(a)

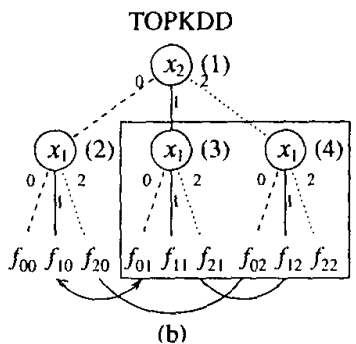

(b)
Figure 6: Sifting of OPKDDs having complemented edges.

\subsection{Sifting Operations on TOPKDDs}

As shown above, sifting levels in a TOPKDD can be done solely by local operations on the diagram. This however is true only in the case where edges occur exclusively between neighboring levels. This is not really a problem (the number of nodes affected by the sifting is still bounded by the number of incoming edges). However, (for the sake of simplicity), we ensure to have edges exclusively between neighboring levels. That is, the diagram is quasi reduced, having cross-point nodes $f$, iff $f_{0}=f_{1}$. Furthermore we assume all functions are rooted at the top level. In order to device a sifting-based dynamic reordering algorithm for OPKDDs, let us first consider a number of special cases, exemplified below.

Example 3 Consider the TOPKDDs shown in Figure 5, (under the assumption that the diagram quasi reduced). The lower nodes in (a) have a dashed outline, and represent crosspoint nodes, i.e., mere connections to the next level in the diagram. After sifting levels, we obtain the OPKDD $(b)$, having a single cross-point node. Although it might look like we have done a reduction of the TOPKDD this is not really the case. A fully reduced TOPKDD will have exactly the same number of nodes. The complementary situation is obtained by reversing the procedure.

From the above example it is obvious that we will have to keep track of the number of "real" nodes (i.e., non cross-point nodes) in the TOPKDD.

\subsection{Sifting of TOPKDDs having Complemented Edges}

To be able to utilize complemented edges we need to ensure that canonicity is preserved by the local variable exchange dynamic_reordering \{

1 compute initial cost

2 for each variable; find initial position

3 sift towards top; keep track of best position

4 sift all way to bottom; keep track of best position

5 apply best position; repeat 2 until no further improvement \}

Figure 7: Dynamic reordering of TOPKDDs.

operation. Let us start by making the following observation; nodes above and below the levels sifted are unaffected by the level exchange operation, i.e., the cofactors below remains intact.

Therefore, we need only to consider the canonicity criteria for the two levels about to be sifted. Assume that the TOPKDD shown in Figure 6 (a) is canonical. After the level exchange, node (2) is still canonical, as canonicity is defined from the polarity of the 0 -edge. The edge to $f_{00}$ is un-complemented (positive), since the initial TOPKDD (a) is canonical, thus node (2) is also canonical. Now consider nodes (3) and (4). In these cases we have to apply the canonicity criteria, as their 0 -edge polarities might change (edges pointing to $f_{01}$ and $f_{02}$ respectively. For node (1) this implies possible polarity changes only on the 1-edge and the 2-edge, thus also node (1) will remain canonical.

By this we have shown that sifting levels in a TOPKDD having completed edges is a truly local operation and can be performed solely by the redirection of edges together with some possible edge polarity changes.

\subsection{Dynamic Reordering of TOPKDDs}

The best known algorithms for finding the optimal order for non-symmetric DDs all have exponential worst-case behavior in the number of input variables, see e.g. $[9,6,11]$. Therefore, we have to settle for heuristic approaches. Utilizing the properties shown in Sections 3, 3.2 and 3.3, we have developed a dynamic reordering algorithm similar to $[10,16]$. The algorithm is simple, iteratively sifting each variable to the position where the number of real TOPKDD nodes is minimized. The iteration is aborted when no further improvement is obtained. Our algorithm is outlined in Figure 7.

We have also tried a slightly different strategy. By greedily choosing the variable (to sift) corresponding to the level having largest number of real nodes. However, our experimental results have shown the above simple algorithm to produce the best results. Based on the property of local variable exchange more sophisticated reordering techniques known for OBDDs can be directly transferred $[15,13,14,20]$. 


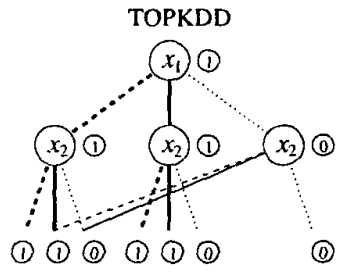

(a)

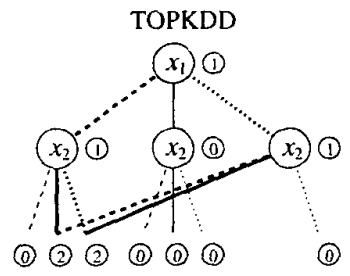

(b)
Figure 8: TOPKDD-based minimization.

\section{HEURISTIC MINIMIZATION OF OPKDDS}

As shown in Section 3 a TOPKDD implicitly holds all possible OPKDD representations under a given variable order (including both the worst and best representation). The number of possible representations (under a given order) is $O\left(3^{|G|}\right)(|G|$ being the number of nodes in the reduced TOPKDD) making exhaustive methods intractable.

Choosing decomposition type for a node activates two out of three outgoing edges (cross-point nodes used for connecting levels can be considered to have only one outgoing edge). Only nodes on active paths are part of the OPKDD encoded in the TOPKDD. Figure 8 show examples of TOPKDDs encoding OPKDDs. Active edges are marked with fat lines. Keeping track of active nodes are done by the use of reference counters (shown as circled numbers in the figure), accounting the number of active incoming edges for each node. By this, we can change the decomposition type of any node in the TOPKDD while updating the number of OPKDD nodes required. If the reference counter for a node $g$ is decreased to 0 , visit the active successors (according to decomposition type) of $g$ and decrease their values by 1 . The complementary situation occurs if a counter value is increased from 0 to 1 , in which case we have to visit the corresponding active successors increasing their value by 1 . (Both increasing and decreasing reference counters might cause a recursive traversal of the TOPKDD.)

We have developed a heuristic minimization method featuring a parameterized look-ahead technique. A window can be set defining the scope (upper, and lower diagram level limits) in which we explore different decomposition types. Furthermore, the recursion depth of the look-ahead can be selected. E.g., set to a depth of $d$ will cause the decomposition types of any combination of $d$ nodes within the scope to be simultaneously considered. The method is greedy in that once a reduction is obtained we apply the corresponding decomposition. We repeat the procedure until no further improvements are obtained. In order to reduce the complexity our method considers only the decomposition types for nodes currently active within the scope.

Figure 8 (a) shows an initial solution (encoding an OBDD), having 7 active nodes (i.e., nodes having, non-0 reference counters). After applying the minimization we obtain an OP-
KDD having only 5 nodes. Note, by a simple approach without look-ahead, the reduction obtained by assigning a $p D$ decomposition to the top node could not be foreseen. Our method is somewhat similar to the one presented in [18], but to the best of our knowledge, both the the scoping and lookahead technique is novel.

As a starting point we can choose any initial DD representation, e.g., OBDD, OKFDD or OPKDD.

\subsection{Experimental Results}

Our algorithms are programmed in a prototype package, utilizing the CUDD 2.3.0 [21] package for OBDD operations. Custom data types are used to represent TOPKDD and OPKDD nodes. Complemented edges are used for both OBDDs and TOPKDDs. The max number of nodes at one level is set to 1000 . The size of DDs is measured by the number of non-terminal nodes.

The first experiment, Table 1, shows the effect of dynamic reordering on TOPKDDs. Left columns show number of nodes starting from a naive variable ordering (as given by the initial PLA benchmarks), while right columns show the number of nodes starting out from a minimized OBDD. For the OBDD minimization we have applied the "group sifting with convergence", as implemented in the CUDD package. Numbers in "( )", show the size of minimized OPKDDs starting from the corresponding TOPKDD, as further discussed in the final experiment below.

Our experimental results show great improvement to the naive ordering, e.g., almost 15 times node reduction for "add6". We observe that a minimized OBDD (having a suitable OBDD variable order), often provides a plausible starting point for dynamic TOPKDD reordering.

The second experiment (shown in Table 2), gives a comparison of the heuristic minimization method for OPKDDs proposed in Section 4. Column $O B D D$ gives the number of minimized OBDD nodes using CUDD "group sifting with convergence". Column $O P K D D$ shows the results from our TOPKDD-based minimization method under the ordering given by the minimized OBDD. For the experiment, we have applied two different parameter settings. To the left, both the scope size and recursion depth are set to 1. I.e., for each iteration, nodes belonging to a single level is considered individually. To the right, scope size and recursion depth are set to 2. For each iteration two neighboring levels are considered, (scope set to 2), and decomposition types are pairwise selected, (recursion depth set to 2). Columns OKFDD [5] and $O P K D D$ [18], present other state of the art results. (The OKFDD results were obtained on the PLA benchmarks suite, and differ in cases from the results presented in [5] based on the BLIF benchmarks.) Numbers in "( )" show the initial OBDD size. As the approach from [18] does not utilize complemented edges, the number of nodes should be at least as large as in column $O B D D$. This is not always the case. However, if don' $t$ care information of the functions is utilized 
to minimize the initial OBDDs, the numbers presented may prove to be correct. Unfortunately, we cannot claim to make a fair comparison to the results from [18].

This experiment confirms our expectations of OPKDD compactness, compared to the initial OBDDs. The OKFDDs [5], are derived by a genetic algorithm, simultaneously seeking suitable ordering and decomposition types. The quality of the results are excellent, see e.g. "apex7" and "duke2", at the expense of CPU resources. However, even though our approach $O P K D D$ does not consider variable reordering, the flexibility of OPKDD representation allows better solutions in some cases, e.g., "bw", "clip" and "tial", while being magnitudes faster.

In the third set of experiments (shown in Table 3), we show the effect of starting point for OPKDD minimization. Once again, column $O B D D$ gives the number of minimized OBDD nodes using CUDD "group sifting with convergence". Column $O K F D D$ [4] gives the number of nodes after siftingbased reordering, [4]. For the OPKDD minimization, scope and recursion depth are set to 2. The derived OPKDDs show significant improvements to their initial representations in most cases. In Table 1 we derive OPKDDs starting from minimized TOPKDDs (numbers shown in"( )"). Also in this case we can improve on the initial DD sizes. We conclude that finding a good starting point for OPKDD minimization is crucial to the quality of the final result.

\section{CONCLUSIONS}

In this paper we have investigated a number of important properties of Ternary-OPKDDs and OPKDDs. The conditions under which TOPKDDs having complemented edges offer a canonical representation are given. Furthermore, we have shown that sifting operations on TOPKDDs (with or without complemented edges) are truly local and can be pursued solely by the redirection of edges. These properties are utilized by new heuristic minimization methods, both for TOPKDD and OPKDDs. The efficiency of the proposed heuristics is demonstrated on a set of MCNC benchmarks and confirm the potential compactness of OPKDDs for bit-level representation of switching functions.

The implicit multi-level representation of the DD may be utilized directly or as a starting point for finding decomposition trees. Therefore, a future research direction is to investigated whether the size reduction also corresponds to an area reduction in multi-level synthesis.

\section{REFERENCES}

[1] B. Becker and R. Drechsler. How many decomposition types do we need? In European Design \& Test Conf., pages $438-443,1995$.

\begin{tabular}{|l|r|r|r|r|r|}
\hline name & in/out & OBDD & OPKDD & OKFDD I5 & OPKDD II8] \\
\hline 5xp1 & $7 / 10$ & 41 & $32 / 28$ & 28 & $33(68)$ \\
9 sym & $9 / 1$ & 24 & $24 / 24$ & 24 & $26(33)$ \\
add6 & $12 / 7$ & 28 & $23 / 23$ & 23 & $23(47)$ \\
alu2 & $10 / 6$ & 86 & $81 / 75$ & 68 & $59(61)$ \\
apla & $10 / 12$ & 105 & $96 / 94$ & 105 & \\
apex7 & $48 / 37$ & 289 & $271 / 268$ & 186 & - \\
bc0 & $26 / 11$ & 522 & $474 / 469$ & 424 & \\
bw & $5 / 28$ & 97 & $86 / 83$ & 91 & $90(108)$ \\
chkn & $29 / 7$ & 267 & $255 / 255$ & 242 & \\
clip & $9 / 5$ & 74 & $66 / 64$ & 72 & $69(97)$ \\
col4 & $14 / 1$ & 26 & $26 / 26$ & 26 & $26(27)$ \\
con1 & $7 / 2$ & 17 & $14 / 14$ & 13 & $14(15)$ \\
dc2 & $8 / 7$ & 61 & $56 / 55$ & 61 & $54(64)$ \\
dist & $8 / 5$ & 120 & $112 / 109$ & 120 & $127(152)$ \\
dk17 & $10 / 11$ & 92 & $89 / 85$ & 84 & $62(63)$ \\
dk27 & $8 / 9$ & 61 & $56 / 56$ & 54 & $25(22)$ \\
duke2 & $22 / 29$ & 355 & $341 / 338$ & 286 & $269(373)$ \\
exp & $8 / 18$ & 169 & $149 / 144$ & 158 & \\
f51m & $8 / 8$ & 38 & $30 / 26$ & 25 & $28(67)$ \\
in2 & $19 / 10$ & 234 & $191 / 187$ & 171 & - \\
in7 & $26 / 10$ & 79 & $77 / 77$ & 67 & \\
inc & $7 / 9$ & 70 & $66 / 65$ & 67 & $58(70)$ \\
intb & $15 / 7$ & 537 & $411 / 386$ & 385 & - \\
misex1 & $8 / 7$ & 35 & $33 / 32$ & 32 & $31(36)$ \\
misex3 & $14 / 14$ & 520 & $481 / 467$ & 484 & \\
misj & $35 / 14$ & 39 & $39 / 39$ & 40 & $43(43)$ \\
mlp4 & $8 / 8$ & 134 & $107 / 103$ & 107 & $99(141)$ \\
rd53 & $5 / 3$ & 16 & $13 / 13$ & 13 & $13(23)$ \\
rd73 & $7 / 3$ & 30 & $26 / 25$ & 21 & $21(43)$ \\
rd84 & $8 / 4$ & 41 & $36 / 46$ & 29 & $29(59)$ \\
risc & $8 / 31$ & 65 & $60 / 57$ & 56 & $57(67)$ \\
pdc & $16 / 40$ & 602 & $542 / 538$ & 564 & \\
sao2 & $10 / 4$ & 80 & $69 / 66$ & 79 & $69(85)$ \\
sex & $9 / 14$ & 41 & $38 / 38$ & 37 & $38(47)$ \\
spla & $16 / 46$ & 590 & $526 / 525$ & 562 & \\
t481 & $16 / 1$ & 20 & $18 / 18$ & 16 & $17(32)$ \\
tial & $14 / 8$ & 579 & $362 / 357$ & 474 & $381(690)$ \\
ts10 & $22 / 16$ & 165 & $165 / 165$ & 123 & $132(142)$ \\
vg2 & $25 / 8$ & 80 & $80 / 77$ & 83 & \\
x6dn & $39 / 5$ & 240 & $215 / 213$ & 210 & 5 \\
xor5 & $7 / 10$ & 5 & $5 / 5$ & 5 & - \\
\hline
\end{tabular}

Table 2: OPKDD minimization.

\begin{tabular}{|l|r|r|r|r|r|}
\hline & & \multicolumn{2}{|c|}{ OBDD Order } & \multicolumn{2}{|c|}{ OKFDD Order } \\
name & in/out & $O B D D$ & $O P K D D$ & $O K F D D[4]$ & $O P K D D$ \\
\hline 5xp1 & $7 / 10$ & 41 & 31 & 28 & 28 \\
add6 & $12 / 7$ & 28 & 23 & 23 & 23 \\
bc0 & $26 / 11$ & 523 & 469 & 431 & 412 \\
duke2 & $22 / 29$ & 355 & 338 & 300 & 274 \\
exp & $8 / 18$ & 170 & 147 & 158 & 134 \\
in2 & $19 / 10$ & 234 & 187 & 164 & 147 \\
in7 & $26 / 10$ & 79 & 77 & 64 & 64 \\
inc & $7 / 9$ & 70 & 65 & 66 & 62 \\
intb & $15 / 7$ & 538 & 386 & 480 & 366 \\
misex3 & $14 / 14$ & 520 & 426 & 583 & 491 \\
sao2 & $10 / 4$ & 80 & 68 & 80 & 73 \\
tial & $14 / 8$ & 580 & 359 & 550 & 359 \\
vg2 & $25 / 8$ & 80 & 80 & 175 & 164 \\
x6dn & $39 / 5$ & 241 & 213 & 215 & 202 \\
\hline
\end{tabular}

Table 3: OPKDD minimization from different orderings. 


\begin{tabular}{|l|r|r|r|r|r|r|r|}
\hline & \multicolumn{3}{|c|}{ Naive Ordering } & \multicolumn{3}{|c|}{ Minimized OBDD } \\
name & in/out & OBDD & TOPKDD org & TOPKDD dyn & OBDD & TOPKDD org & TOPKDD dyn \\
\hline 5xp1 & $7 / 10$ & 74 & 146 & $48(31)$ & 42 & 49 & $48(31)$ \\
add6 & $12 / 7$ & 309 & 1440 & $103(62)$ & 29 & 28 & $28(23)$ \\
exp & $8 / 18$ & 210 & 571 & $422(177)$ & 170 & 454 & $370(134)$ \\
in2 & $19 / 10$ & 2361 & - & - & 235 & 1015 & $760(176)$ \\
in7 & $26 / 10$ & 235 & 701 & $177(84)$ & 80 & 265 & $144(71)$ \\
inc & $7 / 9$ & 77 & 158 & $136(63)$ & 71 & 167 & $144(62)$ \\
sao2 & $10 / 4$ & 155 & 428 & $185(69)$ & 81 & 186 & $185(69)$ \\
\hline
\end{tabular}

Table 1: TOPKDD dynamic reordering.

[2] K.S. Brace, R.L. Rudell, and R.E. Bryant. Efficient implementation of a BDD package. In Design Automation Conf., pages 40-45, 1990.

[3] R.E. Bryant. Graph - based algorithms for Boolean function manipulation. IEEE Trans. on Comp., 35(8):677-691, 1986.

[4] R. Drechsler and B. Becker. - Ordered Kronecker functional decision diagrams - a data structure for representation and manipulation of boolean functions. IEEE Trans. on CAD, 17(10):965-973, 1998.

[5] R. Drechsler, B. Becker, and N. Drechsler. OKFDD minimization by genetic algorithms with application to circuit design. INTEGRATION, the VLSI Jour., 28(2):121-139, 1999.

[6] R. Drechsler, N. Drechsler, and W. Günther. Fast exact minimization of BDDs. In Design Automation Conf., pages 200-205, 1998.

[7] R. Drechsler and W. Günther. Using lower bounds during dynamic BDD minimization. In Design Automation Conf., pages 29-32, 1999.

[8] R. Drechsler, A. Sarabi, M. Theobald, B. Becker, and M.A. Perkowski. Efficient representation and manipulation of switching functions based on ordered Kronecker functional decision diagrams. In Design Automation Conf., pages 415-419, 1994.

[9] S.J. Friedman and K.J. Supowit. Finding the optimal variable ordering for binary decision diagrams. In $\mathrm{De}$ sign Automation Conf., pages 348-356, 1987.

[10] M. Fujita, Y. Matsunaga, and T. Kakuda. On variable ordering of binary decision diagrams for the application of multi-level synthesis. In European Conf. on Design Automation, pages 50-54, 1991.

[11] W. Günther and R. Drechsler. Minimization of free BDDs. In ASP Design Automation Conf., pages 323326, 1999.

[12] S. Minato, N. Ishiura, and S. Yajima. Shared binary decision diagrams with attributed edges for efficient
Boolean function manipulation. In Design Automation Conf., pages 52-57, 1990.

[13] D. Möller, P. Molitor, and R. Drechsler. Symmetry based variable ordering for ROBDDs. IFIP Workshop on Logic and Architecture Synthesis, Grenoble, pages 47-53, 1994.

[14] S. Panda and F. Somenzi. Who are the variables in your neighborhood. In Int'l Conf. on CAD, pages 74-77, 1995.

[15] S. Panda, F. Somenzi, and B.F. Plessier. Symmetry detection and dynamic variable ordering of decision diagrams. In Int'l Conf. on CAD, pages 628-631, 1994.

[16] R. Rudell. Dynamic variable ordering for ordered binary decision diagrams. In Int'l Conf. on CAD, pàges $42-47$, 1993.

[17] T. Sasao. Representations of logic functions using EXOR operations. In T. Sasao and M. Fujita, editors, Representation of Discrete Functions, pages 2954. Kluwer Academic Publisher, 1996.

[18] T. Sasao and J. Butler. A design method for look-up table type FPGA by pseudo-Kronecker expansion. In Int'l Symp. on Multi-Valued Logic, pages 97-106, 1994.

[19] C. Scholl and B. Becker. On the generation of multiplexer circuits for pass transistor logic. In Design, Automation and Test in Europe, 2000.

[20] C. Scholl, D. Möller, P. Molitor, and R. Drechsler. BDD minimization using symmetries. IEEE Trans. on CAD, 18(2):81-100, 1999.

[21] F. Somenzi. CUDD: CU Decision Diagram Package Release 2.3.0. University of Colorado at Boulder, 1998.

[22] C.C. Tsai and M. Marek-Sadowska. Multilevel logic synthesis for arithmetic functions. In Design Automation Conf., pages 242-247, 1996. 